\title{
¿QUIEN ESTUDIA CIENCIA EN NUESTRA UNIVERSIDAD?
}

\author{
ESCUDERO ESCORZA, T. \\ ICE Universidad de Zaragoxa.
}

\begin{abstract}
SUMMARY
The analysis of psychological and educational initial characteristics of students of science and technology in the Spanish University --in contrast with the characteristics of students of other fields - and the relationship with later achievement, is the purpose of the present article. Comparisons between male and female students ara also included.
\end{abstract}

\section{INTRODUCCIÓN}

Es bien conocido por los estudiosos y por los planifícadores de la educación el descenso relativo de alumnos en las carreras cientifico-tecnológicas que ha tenido lugar en las últimas décadas. Esta situación, en unión de otros aspectos como disminución de profesorado, descenso en rendimientos, etc. ha dado lugar a que determinados autores sigan hablando de crisis en la enseñanza de las ciencias y, al mismo tiempo, ofrezcan análisis de tipo diagnóstico del problema (Yager y Penick 1987).

Estos problemas que las revistas especializadas nos sitúan en otros contextos educativos existen igualmente en nuestro sistema educativo, donde la masificación universitaria no se produce en ciencias y donde los alumnos parecen acercarse cada vez en menor proporción al estudio de la ciencia.

El presente trabajo, sin entrar directamente en el análisis de esta hipotética crisis, se inscribe en su entorno, puesto que intenta ofrecer el perfil psico-educativo de los alumnos que eligen ciencias como área de estudio, en contraste con el de los alumnos que no lo hacen. Aparte de otras incidencias de tipo propiamente didáctico, no existe duda de que el problema que analizamos tiene una relación directa con uno de los factores determinantes de la denominada crisis, esto es, la escasez relativa de alumnos en las carreras cientificotecnológicas en nuestra Universidad.

\section{EL ESTADO DE LA CUESTIÓN}

Las caracteristicas de los alumnos son, sin lugar a dudas, parte importante del contexto (antecedentes) que, como señala Welch (1985), junto con las transacciones (interacciones) y los rendimientos académicos cons- tituyen los tres principales componentes del dominio de la enseñanza de las ciencias. Las aptitudes, las destrezas, los intereses, los rasgos de personalidad, etc. de Ios alumnos han sido y siguen siendo motivo de estudio y reflexión para los didactas de las ciencias, entre otras razones porque son claros condicionantes de los resultados del aprendizaje, a través de la interacción didáctica.

En coherencia con esta línea argumental se mueve Lowery (1980) al construir su modelo organizativo de la investigación en didáctica de las ciencias. La segunda fase del modelo - tras la recolección básica y previamente a la formulación teórica - se asienta en tres círculos entrelazados que representan al que aprende, a la instrucción y a las ciencias. De las condiciones de cada uno de estos componentes (círculos) y de las interacciones entre ellos, se derivará una mejor o peor educación cientifica.

Aưnque la investigación especializada (Stinkamp y Maerh 1983, Collings y Smithers 1984) ha centrado muchos esfuerzos y obtenido abundantes y consistentes resultados en el estudio de las características de los estudiantes de ciencias y de sus relaciones con sus rendimientos académicos, todavía existen muchos problemas por resolver en tales áreas, que tienen carácter prioritario (Butts et al. 1978, Abraham et al., 1982) y que, al mismo tiempo, son punto de referencia inicial para varias líneas de investigación consideradas por los especialistas como las más relevantes e importantes para el desarrolio de la enseñanza de las ciencias (Welch 1985).

¿Que factores condicionan y determinan las actitudes ante la ciencia? ¿Cuáles son los obstáculos principales para aprender ciencia? ¿Qué es lo que determina la elec- 
ción o no de estudios científicos?... Estas y otras cuestiones solamente podrán responderse adecuadamente profundizando más, entre otros aspectos, en el conocimiento de las características de los estudiantes y sus relaciones con el aprendizaje. Todavía existen algunas deficiencias en cuanto a recolección de datos básicos de identificación, pero se hace cada vez más necesario el desarrollo de modelos de organización que permitan comparar paradigmas y separar lo relevante de los irrelevante y, finalmente, intentar articular eficazmente la fundamentación teórica del aprendizaje de las ciencias (Lowery 1980).

El conocimiento de las citadas características de los estudiantes de ciencia y de sus relaciones se hace verdaderamente critico a partir de la educación secundaria, que es cuando las asignaturas de ciencias se convierten progresivamente en opcionales. El problema adquiere todavía más relevancia a la vista de la creciente tendencia entre los estudiantes, a no elegir opciones científicas (Weich 1985), o de la escasa representación femenina entre los alumnos de determinados estudios científico-tecnológicos (Benbow y Minor 1986, Ethington y Wolfle 1986, Deboer 1986).

La teoría ocupaciones de Holland (1973) viene a indicar que todo individuo tiende a elegir y situarse académica y profesionalmente en función de sus actitudes, intereses, valores, tipo de personalidad y habilidades. En otras palabras, si las características de un individuo concuerdan con las exigencias y las caracteristicas de una determinada ocupación profesional, aquél la elegirá y tendrá éxito en el aprendizaje de las destrezas asociadas con tal ocupación. Cuando los estudiantes no eligen ciertas disciplinas o rinden poco en ellas, debemos pensar por tanto, que sus características personales no se corresponden con las requeridas por tales disciplinas.

Apoyándonos en estos planteamientos teóricos de HoIland y razonando a la inversa, habremos de convenir que las características de los estudiantes que eligen cursos o carreras de ciencias, deben ser una buena aproximación, en conjunto, de los rasgos de los individuos que facilitan el aprendizaje científico. Pues bien, la investigación viene a decirnos que comparándolos con los estudiantes que eligen otro tipo de estudios, los alumnos de ciencias, en general, poseen ur coeficiente intelectual alto, con dominio del pensamiento convergente sobre el divergente, con buena habilidad espacial y matemática, con tendencia a la introversión, al orden y a la apreciación estética del mundo, con baja ansiedad, con escasa orientación hacia los aspectos personales y sociales, con buena actitud hacia la ciencia y, obviamente, con buen autoconcepto sobre su capacidad para el aprendizaje científico (Collings y Smithers 1984, Baker 1985).

Recientemente se ha redoblado el esfuerzo investigador en el estudio de las diferencias que se producen en la enseñanza de las ciencias en razón del sexo, así co- mo de las causas y de las consecuencias de tales diferencias. El alejamiento progresivo de las alumnas, de la física, por ejemplo, se ha intentado explicar como consecuencia de una mayor «orientación hacia las personas» que "hacia las ideas y las cosas» en las mujeres que en los varones (Collings y Smithers 1984), o como resultado del menor autoconcepto como estudiante de ciencias demostrado por las alumnas, con independencia de su mayor o menor rendimiento académico (DeBoer 1986), o como consecuencia de un proceso de interacción no aditivo del sexo con factores predictores del aprendizaje, de manera desventajosa para las mujeres (Ethington y Wolfle 1986), o como efecto de las diferencias sexuales en relación con las aptitudes espacial y verbal (Hacker 1986) y el razonamiento matemático (Benbow y Minor 1986).

En general, la búsqueda de explicaciones a fenómenos relativos al aprendizaje científico y al sexo se hace desde perspectivas muy diversas, dentro del rango que existe entre planteamientos fundamentales ambientalistas y otros básicamente biologistas (Hacker 1986). De cualquier manera, los resultados de la investigación no perfilan un marco nítido y concluyente como para pensar que ya no es necesario seguir investigando sobre estos iemas; todavía existen muchas lagunas y bastantes imprecisiones.

En cuanto a las relaciones de las diversas caracteristicas personales con el rendimiento académico, las sintesis de resultados señalan que las diferencias por sexo parecen ser escasas y que las habilidades previas sueIen mostrar una mayor relación con el rendimiento que las actitudes (Steinkamp y Maehr 1983, Weich 1985, Escudero 1985).

\section{CARACTERISTICAS DE LOS ESTUDIAN- TES EN NUESTRO SISTEMA EDUCATIVO}

En un reciente estudio de seguimiento de una cohorte de alumnos desde el COU hasta el final de su carrera universitaria, para analizar la capacidad predictiva de nuestro examen de selectividad (Escudero 1986), analizamos las caracteristicas de los alumnos en un amplio número de variables de rendimiento, de aptitud y de personalidad. La muestra era de 417 alumnos distribuidos por los diferentes tipos de estudios y centros de las Universidades de Navarra y Zaragoza.

En el presente trabajo presentamos un nuevo análisis de datos del citado estudio de seguimiento, en la línea de investigación que hemos descrito en el apartado anterior. En concreto, comparamos las características de los alumnos de ciencias con el resto de los estudiantes, con análisis diferenciados por sexo. Incluimos, asimismo, el estudio para cada grupo de alumnos de las correlaciones de tales características con el rendimiento global a lo largo de todos los estudios universitarios.

Las caracteristicas analizadas en los estudiantes son: 
a) Medidos comunes de preparación previa: 1) primer ejercicio del examen de acceso (conferencia + análisis de texto), 2) segundo ejercicio del examen de acceso (parte común), 3) expediente secundario, 4) prueba de madurez en asignaturas comunes de BUP, 5) prueba de madurez en asignaturas comunes de COU, y 6 ) prueba global de madurez en asignaturas comunes.

b) Aptitudes: 1) coeficiente de inteligencia (D-48), 2) comprensión verbal (B.V.-17), 3) razonamiento numérico (DAT-NA), 4) razonamiento abstracto (DAT-AR), y 5) atención (DAT-CSA).

c) Rasgas de personalidad (inventario de Bernreuter): 1) inestabilidad emocional (B1-N), 2) autosuficiencia (B2-S), 3) introversión-extraversión (B3-I), 4) dominiosumisión (B4-D), 5) confianza en sí mismo ( $\mathrm{Fl}-\mathrm{C})$, y 6) sociabilidad (F2-S).

El análisis de los valores promedio, para varones y mujeres, de estas características lo hemos hecho para los alumnos de la rama de ciencias y letras en BUP, para los alumnos de carreras de ciencias, humanidades, medicina, ingeniería industrial y magisterio. La significación estadística en la comparación de medias la hemos analizado con la ayuda de la aproximación de WelchAspin del test $t$ (Marascuilo 1971, apartados 11.9 y 13.3).

Los resuitados empiricos son recogidos en tablas y comentados a continuación. Por razones de espacio y re- levancia, nos limitamos a reproducir los datos y resultados que nos parecen más representativos.

\subsection{Preparación académica previa a la Universidad}

Como ya se ha indicado, en el bloque de preparación previa a la Universidad consideramos los dos ejercicios comunes del examen de acceso, el expediente secundario y tres medias - una de ellas promedio de las otras dos- de madurez académica previa. La Tabla I recoge los parámetros estimados para diversos grupos de alumnos.

En Ingeniería nos limitamos a la muestra de varones, puesto que la representación femenina es muy pequeña.

Obviamente, no tiene mucho sentido reproducir el nivel de significación estadística de las múltiples comparaciones de medias en todas las variables, basta con que resaltemos aquellos valores extremos en ambos sentidos que tienen más interés para el propósito de nuestro trabajo.

En el primer ejercicio de la selectividad (conferencia + análisis de texto) resulta curioso que, salvando los varones de Magisterio, son los varones de Ciencias los que ofrecen el promedio más bajo, mientras que las mujeres de Ciencias ofrecen uno de los más altos. En concreto, la significación estadística de la diferencia entre las medias de estos dos últimos se produce al nivel $p<0,02$.

Tabia I.

Medias y desviaciones tipicas muestrales en variables de preparación académica previa a la Universidad.

\begin{tabular}{|c|c|c|c|c|c|c|}
\hline $\begin{array}{c}\text { Muestra } \\
\text { (tamkino muestrai) }\end{array}$ & $\begin{array}{c}1^{\text {er ejercicio }} \\
\text { selectividad }\end{array}$ & $\begin{array}{l}20 \text { ejercicio } \\
\text { selectividad }\end{array}$ & $\begin{array}{l}\text { Expediante } \\
\text { secundario }\end{array}$ & $\begin{array}{l}\text { Madirez } \\
\text { asig.con. gip? }\end{array}$ & $\begin{array}{l}\text { Nadurez } \\
\text { asig.corm..OU }\end{array}$ & $\left\{\begin{array}{l}\text { Medirez gloxal } \\
\text { en asig. cromas }\end{array}\right.$ \\
\hline $\begin{array}{l}\text { Ciencias en BP } \\
\text { Varones }(192) \\
\end{array}$ & 5,$25 ; 1,19$ & 4,$65 ; 1,66$ & 7,$03 ; 1,05$ & $2,83: 0,283$ & $4, \infty ; 1,00$ & $3,2: 0, n$ \\
\hline $\begin{array}{l}\text { Ciercias on filp } \\
\text { Mujeras (15a) }\end{array}$ & 5,$59 ; 0,92$ & 5,$19 ; 1,59$ & $7, \infty: 1,27$ & 2,$08 ; 0.81$ & $4: 1,05$ & $3, \therefore: 0,76$ \\
\hline $\begin{array}{l}\text { Letras en BiR } \\
\text { Varones (24) }\end{array}$ & 5,$59 ; 2,10$ & 4,$72 ; 1,83$ & 7,$32 ; 0,92$ & 2 姆；0,78 & $3,80: 1,15$ & $3,21: 0,70$ \\
\hline $\begin{array}{l}\text { Letras on afp } \\
\text { unjeres (4\%) }\end{array}$ & $5,46: 1,13$ & $4.99 ; 1.73$ & 6,$77 ; 1,33$ & $2.48 ; 0.87$ & 3,$68 ; 2,23$ & 2,$86 ; 0,78$ \\
\hline $\begin{array}{l}\text { Carrera Ciencias } \\
\text { Varones (34) }\end{array}$ & 5,$10 ; 1,24$ & 4,$08 ; 1,52$ & $7,24: 1,13$ & 2,$93 ; 0,98$ & $4, \infty ; 1,09$ & 3,$29 ; 0,89$ \\
\hline $\begin{array}{l}\text { Carrera Ciencias } \\
\text { Mujeres (39) }\end{array}$ & 5,$66 ; 0,77$ & 5,$47 ; 1,62$ & $7,16: 1,42$ & $2,87: 0, \infty$ & 3,$96 ;: .11$ & 3,$23 ; 0,02$ \\
\hline $\begin{array}{l}\text { Carr. Hmenidacies } \\
\text { Varones (25) }\end{array}$ & 5,$69 ; 1,11$ & 4,$57 ; 1,61$ & 7,$34 ; 0,88$ & $2,81: 0,72$ & 3,$74 ; 1,03$ & 3,$14 ; 0,71$ \\
\hline $\begin{array}{l}\text { Cart. Hmanidades } \\
\text { Mejeres (31) }\end{array}$ & 5,$43 ; 1, \infty$ & $5, \infty ; 1,90$ & $7, \infty 6 ; 1,26$ & 2,$87 ; 0,80$ & 3,$89 ; 1,11$ & 3,$16 ; 0,75$ \\
\hline $\begin{array}{l}\text { Vedicina } \\
\text { Varones (63) }\end{array}$ & 5,$33 ; 1,19$ & $\Delta, \infty 0 ; 1,76$ & 6,$97 ; 1,01$ & $2,1: 12 ; 0,94$ & 4,$02 ; 1,24$ & $3,27: 0,86$ \\
\hline $\begin{array}{l}\text { Hedicina } \\
\text { Mijeres (64) }\end{array}$ & 5,$64 ; 1,08$ & 5,$17 ; 1,48$ & 6,$99 ; 1,19$ & $2.51: 0,8$ & 3,$97 ; 1,08$ & $2,99: 0,73$ \\
\hline $\begin{array}{l}\text { Irgonieria } \\
\text { Varones (34) }\end{array}$ & 5,$22 ; 1,29$ & 5,$47 ; 1,62$ & 7,$27 ; 1,04$ & 2,$19 ; 0,66$ & 4,$41 ; 0,80$ & 3,$32 ; 0,57$ \\
\hline $\begin{array}{l}\text { Nagisterio } \\
\text { Varones (20) }\end{array}$ & 5,$03 ; 1$ & 3,$82 ; 1,67$ & 6,$98 ; 0,86$ & 2,$83 ; 0,88$ & 3,$45 ; 0,94$ & 3,$03 ; 0,71$ \\
\hline $\begin{array}{l}\text { Magisterio } \\
\text { Mujeres (57) }\end{array}$ & 5,$89 ; 0,85$ & 4,$99 ; 1,67$ & 6,$75 ; 1,29$ & 2,$47 ; 0,84$ & $3,85: 1,08$ & 2,$91 ; 0,76$ \\
\hline
\end{tabular}


La situación anterior también se produce con el segundo ejercicio (asignaturas comunes) de la selectividad; destaca claramente el alto promedio de las chicas de Ciencias, mientras que el de los varones es mucho más bajo $(p<0,06)$ y similar al de otras muchas submuestras. En general, en esta variable, el promedio de las mujeres supera claramente al de los varones. La excepción se produce en Ingeniería donde los alumnos (casi todos varones) tienen un promedio relativo muy alto en esta variable.

Los datos sobre el expediente secundario no tienen mucho interés, no solamente porque no aparecen diferencias notorias entre las diversas muestras, sino porque el propio expediente tiene un carácter relativo, ya que las asignaturas cursadas por los alumnos en BUP y COU varian considerablemente de unos alumnos a otros. En todo caso, es oportuno señalar la clara superioridad de expedientes de los varones de Letras en BUP, sobre los de las mujeres de esta misma rama.

Los promedios en las variables de madurez académica en asignaturas comunes de BUP y COU son notablemente altos, en términos comparativos, en las muestras de Ciencias e Ingeniería. Al mismo tiempo, se observa que los varones tienden a mostrar promedios al- go más altos que las mujeres en la mayoria de los casos.

En síntesis, podemos hablar de un panorama bastante diverso en cuanto a preparación previa común. En las pruebas de selectividad destaca el alto nivel relativo de las mujeres de Ciencias, pero no asi en el caso de los varones. En cuanto a las medidas de madurez académica común destacan algo los alumnos de Ciencias e Ingeniería, sobre todo los varones. En general, las mujeres destacan sobre los varones en la prueba de acceso, mientras que éstos lo hacen sobre aquéllas en las pruebas de madurez académica global.

\subsection{Aptitudes mentales}

Muy probablemente son las aptitudes mentales las variables en las que aparecen unas diferencias más acusadas entre las diferentes muestras de alumnos, y de manera especial entre los alumnos que se dirigen a estudios de tipo científico-tecnológico y el resto.

La Tabla 2 reproduce las estadisticas de aptitud de diversas muestras para varones y mujeres así como los baremos para ambas poblaciones.

Los datos resaltan'claramente el hecho de que los promedios de aptitud de las muestras de alumnos del ám-

Tabla II

Medias y desviaciones típicas de diversas muestras de alumnos en las variables de aptitud (Los tamaños muestrales son los indicados en la Tabla 1).

\begin{tabular}{|c|c|c|c|c|c|}
\hline kestra & $\begin{array}{l}\text { Inteligercia } \\
\text { general }\end{array}$ & $\begin{array}{l}\text { Comprensión } \\
\text { vertal }\end{array}$ & $\begin{array}{l}\text { Rascrarisiento } \\
\text { numérico }\end{array}$ & $\begin{array}{l}\text { Razonzriento } \\
\text { abstractio }\end{array}$ & Aterción \\
\hline $\begin{array}{l}\text { Ciencias en Bup } \\
\text { Varcnes }\end{array}$ & 32,$7 ; 5,7$ & 78,$9 ; 15,2$ & 29,$2 ; 6,4$ & $40,3: 5,2$ & 66,$2 ; 10,7$ \\
\hline $\begin{array}{l}\text { Ciercias en BUP } \\
\text { Muljeres }\end{array}$ & 32,$7 ; 5,4$ & 7,$7 ; 14,2$ & 27,$3 ; 6,0$ & $39,4: 5,9$ & 64,$0 ; 12,9$ \\
\hline $\begin{array}{l}\text { Letras en } 8 X P \\
\text { Varores }\end{array}$ & 28,$9 ; 5,7$ & $70,4: 19,6$ & $20,8: 7,0$ & 32,$9 ; 8.6$ & $54,4: 17,6$ \\
\hline $\begin{array}{l}\text { Letras en } \$ Q P P \\
\text { Mujeres. }\end{array}$ & 27,$2 ; 5,4$ & $7 a_{+}, 9 ; 13,4$ & 19,$1 ; 5,4$ & $34,3: 6,0$ & 62,$4 ; 10,6$ \\
\hline $\begin{array}{l}\text { Ciencias } \\
\text { Varones }\end{array}$ & 33,$2 ; 5,5$ & $77,4: 16,3$ & 30,$4 ; 6,7$ & 41,$4 ; 5,2$ & 68,$9 ; 9,6$ \\
\hline $\begin{array}{l}\text { Ciencias } \\
\text { mujeres }\end{array}$ & 35,$0 ; 5,8$ & $80,7: 14,3$ & $\chi \xi, 6: 4,0$ & $41.7: 6,3$ & $63,4: 14,9$ \\
\hline $\begin{array}{l}\text { Humenidades } \\
\text { Varcres }\end{array}$ & 28,$9 ; 5,6$ & $\pi, 0 ; 12,5$ & 22,$3 ; 6,8$ & 34,$4 ; 8,3$ & $59.6: 17.5$ \\
\hline $\begin{array}{l}\text { Humenidades } \\
\text { Mujeres }\end{array}$ & 27,$9 ; 4,6$ & 77,$5 ; 13,5$ & 20,$2 ; 6,3$ & 35,$0 ; 7,2$ & 60,$4 ; 9,1$ \\
\hline $\begin{array}{l}\text { Medicina } \\
\text { varcmes }\end{array}$ & 31,$6 ; 5,3$ & $79,3: 17,3$ & 28,$6 ; 6,2$ & 39,$9 ; 4,7$ & 64,$8 ; 10,4$ \\
\hline $\begin{array}{l}\text { Mexicina } \\
\text { Mujeres }\end{array}$ & 32,$0 ; 5,5$ & $74,2: 15,1$ & 25,$7 ; 6,9$ & 39,$4 ; 5,1$ & 65,$5 ; 12,3$ \\
\hline $\begin{array}{l}\text { Ingenieros } \\
\text { Varores }\end{array}$ & 36,$1 ; 4,8$ & 80,$6 ; 14,8$ & 31,$9 ; 4,5$ & 41,$5 ; 4,4$ & 70,$5 ; 9.8$ \\
\hline $\begin{array}{l}\text { Magisterio } \\
\text { Varones }\end{array}$ & $31,0: 5,6$ & $69.7: 11.5$ & $27,2: 7,4$ & $36,5: 8,1$ & 62,$8 ; 10,3$ \\
\hline $\begin{array}{l}\text { Magisterio } \\
\text { Mujeres }\end{array}$ & 30,$5 ; 5,7$ & 70,$6 ; 13,7$ & 24,$3 ; 6,8$ & 37,$4 ; 5,9$ & 63,$2 ; 12,3$ \\
\hline $\begin{array}{l}\text { Bareno } \\
\text { Varcones }\end{array}$ & 30,$45 ; 5,28$ & 78,$93 ; 11,31$ & $24, \infty ; 7,08$ & 36,$13 ; 7,85$ & 64,$91 ; 10, \pi$ \\
\hline $\begin{array}{l}\text { Bareno } \\
\text { Mujeres }\end{array}$ & 27,$44 ; 5,67$ & 78,$47 ; 11,41$ & $20, \infty ; 7,6$ & 31,$68 ; 8,15$ & $65,18: 10,3$ \\
\hline
\end{tabular}




\section{INVESTIGACIÓN Y EXPERIENCIAS DIDÁCTICAS}

bito científico-tecnológico son más elevados que los de los restantes. Lógicamente esta diferencia no es igua! de acusada en todas las variables. La diferencia se hace especialmente significativa $(p<0,01)$ en determinados rasgos y contrastes, pero en otros el nivel de tal signifícación disminuye sensiblemente. En concreto, las mayores diferencias se dan en el razonamiento numérico y las menores en la comprensión verbal.

También se detecta de los datos que, en relación con los baremos poblacionales, las mujeres presentan, en general, mejores promedios de aptitud que los varo. nes. Esto quiere decir que dentro de la población de mujeres el sistema produce una selección relativamente más exigente que la que produce entre los varones con relación a su población. Este hecho se hace quizás más evidente en los estudios de tipo científico, ya que los rasgos promedio de las mujeres que acceden a eltos son en muchos casos superiores a los de sus compañeros varones, no solamente desde un punto de vista relativo al baremo, sino en términos absolutos.

Sintetizando lo anterior, diremos que los alumnos que acceden a estudios científico-tecnológicos son relativa* mente selectos desde el punto de vista de la aptitud, y que esta selección parece ser todavía más rigurosa en el caso de las mujeres.

\subsection{Rasgos de personalidad}

El tercer y último bloque de características personales de los estudiantes que hemos analizado es el de los rasgos de personalidad que, como hemos visto anteriormente, ha sido frecuente motivo de estudio entre los investigadores en enseñanza de las ciencias.

Por razones de facilidad de comprensión trabajamos en este apartado con rasgos de percentil, cuyos promedios para las diferentes muestras reproduce la Tabla 3. Lógicamente, el punto de referencia general seria el valor 50, a partir del que tendriamos que hablar de alta o baja inestabilidad promedio, extraversión o intro. versión, etc.

Empezando por la inestabilidad emocional diremos que, como promedio, ésta tiende a ser alta, aunque de manera moderada, en todas las muestras. Por otra parte, las diferencias que se observan entre las diversas muestras son poco acusadas y no parecen responder a ninguna tendencia sistemática.

Con la autosuficiencia ocurre al contrario que con la inestabilidad emocional, ya que tiende a ser moderadamente baja en bastantes muestras, aunque en algunas es cercana al valor medio. Solamente se salen ligeramente de esta norma los valores de letras en BUP.

Tabla III

Rasgos de percentil promedio en los rasgos de personalidad para diversas muestras de estudiantes (Los tamaños muestrales son los indicados en la Tabla 1).

\begin{tabular}{|c|c|c|c|c|c|c|}
\hline & & Var & iable & & & \\
\hline Muestra & {$\left[\begin{array}{l}\text { Inestabilidid } \\
\text { emocional }\end{array}\right.$} & Autosuficicsc, & $\begin{array}{l}\text { Introversion } \\
\text { Extreversión }\end{array}$ & $\begin{array}{l}\text { Dominio } \\
\text { Sunisión }\end{array}$ & $\begin{array}{l}\text { Confiarza en } \\
\text { sí mismo }\end{array}$ & Sociabilidad \\
\hline $\begin{array}{l}\text { Ciencias en BUP } \\
\text { Varones }\end{array}$ & 66,3 & 37,4 & 67,8 & 39,6 & 64,6 & 51,0 \\
\hline $\begin{array}{l}\text { Ciencias en BUP } \\
\text { Mujercs }\end{array}$ & 61,1 & 45,6 & 63,3 & 49,5 & 70,1 & 63,9 \\
\hline $\begin{array}{l}\text { Letras en BUP } \\
\text { Varones. }\end{array}$ & 63,3 & 56,3 & 65,8 & 48,1 & 59,4 & 66,1 \\
\hline $\begin{array}{l}\text { Letras en BUP } \\
\text { Mujeres. }\end{array}$ & 64,9 & 43,2 & 68,9 & 42,5 & 76,2 & 52,9 \\
\hline $\begin{array}{l}\text { Ciencias } \\
\text { Varones }\end{array}$ & 70,6 & 33,3 & 69,6 & 31,6 & 69,4 & 49,7 \\
\hline $\begin{array}{l}\text { Ciencias } \\
\text { Myjeres }\end{array}$ & 62,1 & 47,8 & 63,5 & 48,5 & 71,2 & 58,8 \\
\hline $\begin{array}{l}\text { Humanidedes } \\
\text { Varmes }\end{array}$ & 67,8 & 45,5 & 70,1 & 40,5 & 65.0 & 54,8 \\
\hline $\begin{array}{l}\text { Humanidades } \\
\text { Mujeres }\end{array}$ & 69,1 & 49,7 & 71,5 & 43,2 & 75,3 & 61,4 \\
\hline $\begin{array}{l}\text { Medicina } \\
\text { Varcnes }\end{array}$ & 72,2 & 31,4 & 72,4 & 31,8 & 70,5 & 51,5 \\
\hline $\begin{array}{l}\text { Medicina } \\
\text { Mujeres }\end{array}$ & 59,2 & 41,1 & 61,1 & 51,4 & 69,7 & 48,0 \\
\hline Ingenierns & 61,1 & 46,6 & 65,1 & 46,7 & 57,6 & 53,3 \\
\hline $\begin{array}{l}\text { Magisterio } \\
\text { Varcres }\end{array}$ & $\$ 4,4$ & 35,2 & 64,2 & 46,3 & 64,6 & 49,5 \\
\hline $\begin{array}{l}\text { Magisterio } \\
\text { Mujeres }\end{array}$ & 62,1 & 42.7 & 64,6 & 44,7 & 72,3 & 49,4 \\
\hline
\end{tabular}


Aunque no en todos los casos, se aprecia un nivel ligeramente menor de autosuficiencia en las muestras de varones que en las de mujeres.

En promedio, todos nuestros alumnos universitarios tienden a ser moderadamente introvertidos y no se observan diferencias sensibles en razón del tipo de estudio o del sexo.

Existe una ligera tendencia a la sumisión en nuestros estudiantes, al menos entre los varones de diversos tipos de estudios. También se observa cierta falta de confianza en sí mismo con carácter general, acentuada en las mujeres de bastantes tipos de estudios.

Por último, diremos que la sociabilidad oscila alrededor del valor medio, tendiendo a ser algo más baja para las mujeres que para los varones en algunas muestras. En todo caso, esta tendencia no se presenta de manera sistemática.

Hay que hacer notar que los promedios de la muestra de Letras en BUP de los varones ofrecen ciertos rasgos de singularidad, debido, seguramente, a razones diversas, cuyo análisis no es relevante en el contexto de este trabajo. En todo caso, la influencia de este hecho en el conjunto de los resultados es minima y no modifica las tendencias resaltadas.

\section{RELACIONES CON EL RENDIMIENDO ACADEMICO UNIVERSITARIO}

La segunda fase de nuestro trabajo recoge el estudio de los coeficientes de correlación de las características personales que acabamos de estudiar con el rendimiento académico universitario global, medido como prome. dio de las calificaciones obtenidas a lo largo de toda la carrera y ponderado por la incidencia de suspensos, retrasos y convocatorias.

A continuación recogemos las correlaciones indicadas en una selección de las muestras de alumnos estudia. das, los niveles de significacion estadística correspondientes (Sachs 1978), y los comentarios sobre el significado de los valores obtenidos.

Las correlaciones del rendimiento universitario con las variables de preparación previa son significativas en muchos casos, tal como demuestran los valores que recoge la Tabla 4. Los tamaños muestrales ya han sido indicados con anterioridad. Las dos últimas columnas recogen las correlaciones que delimitan los niveles de significación $\mathrm{p}<0,05 \mathrm{y}<0,01$, de acuerdo con el tamaño muestral.

Como puede observarse, se produce cierta diversidad en las correlaciones del rendimiento universitario con

Tabla IV

Corrclaciones (indice $\times 100$ ) del rendimiento académico universitario global con las variables de preparación academica previa.

\begin{tabular}{|c|c|c|c|c|c|c|c|c|}
\hline Mestra. & $\mid \begin{array}{l}1^{e r} \text { ejurc. } \\
\text { selertividiad }\end{array}$ & $\mid \begin{array}{l}20 \text { e ejerc. } \\
\text { selectividad }\end{array}$ & $\begin{array}{l}\text { Expediente } \\
\text { secindario }\end{array}$ & $\begin{array}{l}\text { Madurez asitg. } \\
\text { contros BLP }\end{array}$ & $\begin{array}{l}\text { Machurez asig. } \\
\text { conumes oal }\end{array}$ & 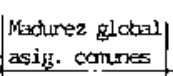 & $\mathrm{P} \quad 0,06$ & P $\quad 0,0$ \\
\hline $\begin{array}{l}\text { Ciencias en BJP } \\
\text { Varones }\end{array}$ & 18 & -03 & 31 & $\infty$ & $\infty$ & $\not 6$ & 14 & 18 \\
\hline $\begin{array}{l}\text { Ciencies an BuPP } \\
\text { Mujeres }\end{array}$ & 11 & 26 & 42 & 23 & 26 & 29 & 16 & ?1 \\
\hline $\begin{array}{l}\text { Letras en BUP } \\
\text { Varnes }\end{array}$ & 53 & $\Delta 2$ & 50 & 42 & 54 & $Q 2$ & 30 & 50 \\
\hline $\begin{array}{l}\text { Letras en BUP } \\
\text { Mjeres. }\end{array}$ & 16 & .33 & 18 & 40 & 28 & AI & 27 & 35 \\
\hline $\begin{array}{l}\text { Cienciass } \\
\text { Varcnes }\end{array}$ & 2? & 05 & 4] & 55 & 43 & 57 & 33 & 42 \\
\hline $\begin{array}{l}\text { Cierxidas } \\
\text { Mujeres }\end{array}$ & xo & 34 & 30 & 23 & 24 & 26 & 30 & 39 \\
\hline $\begin{array}{l}\text { Hutaridades } \\
\text { Varones }\end{array}$ & 36 & 41 & 45 & 59 & 45 & 63 & 38 & 49 \\
\hline $\begin{array}{l}\text { Husmaridates } \\
\text { Mujerns }\end{array}$ & $\alpha B$ & 40 & 54 & 59 & 39 & 60 & 35 & 45 \\
\hline $\begin{array}{l}\text { Medicina } \\
\text { Varones }\end{array}$ & 14 & -17 & 30 & 25 & $Q 2$ & 19 & 25 & 32 \\
\hline $\begin{array}{l}\text { Medicina } \\
\text { Mejeres }\end{array}$ & 20 & 28 & 55 & 37 & 29 & 41 & 24 & $3 ?$ \\
\hline $\begin{array}{l}\text { Ingenieras } \\
\text { Varones }\end{array}$ & 39 & $\infty$ & 43 & 43 & 27 & 48 & 33 & 42 \\
\hline $\begin{array}{l}\text { Magisterio io } \\
\text { Varnoes }\end{array}$ & -11 & 43 & 46 & -38 & 17 & -25 & 42 & 54 \\
\hline $\begin{array}{l}\text { Pagisterio } \\
\text { itujerns }\end{array}$ & 19 & $\Delta l$ & 39 & 30 & 42 & 43 & 26 & 33 \\
\hline
\end{tabular}


la preparación previa, pero éstas son significativas en muchos casos. El expediente y la madurez común correlacionan con el rendimiento universitario de manera más sistemática y con valores más altos que los ejercicios comunes de la selectividad.

Los datos no permiten plantear la hipótesis de que este panorama correlacional es diferente en las carreras de tipo cientifico que en el resto. Lo que si permiten es señalar que parecen existir diferencias entre varones y mujeres, pero este hecho también se observa en otras carreras, además de las científicas. Quizás esta diferencia por sexos tenga diferente orientación en los estudiantes de Ciencias que en los de otro tipo, pero resulta muy aventurado ir más allá en esta discusión.

Las aptitudes medidas no correlacionan significativamente con el rendimiento promedio en la mayoría de los casos estudiados; Ia inteligencia general y el razonamiento general, en ninguna muestra, la atención solamente en la de Magisterio-Varones $(\mathrm{p}<0,05)$ y la comprensión verbal y el razonamiento numérico, en tres casos.

La comprensión verbal correlaciona significativamente con el rendimiento universitario global en las muestras de Letras en BUP-Varones ( $p<0,01)$, CienciasVarones $(p<0,05)$ y Humanidades-Varones $(p<0,01)$. El razonamiento numérico to hace significativamente en el caso de los de Letras en BUP-Varones $(p<0,05)$, Humanidades-Mujeres $(p<0,05)$ y Medicina-Mujeres $(p<0,01)$.

Quizás podría pensarse que la comprensión verbal aparece como un predictor significativo del rendimiento universitario en los varones, mientras que el razonamiento numérico lo hace en el caso de las mujeres.

Con los rasgos de personalidad no puede hablarse de correlaciones significativas con el rendimiento académico universitario global. Se confirma que estas variables son linealmente independientes, aunque los rasgos de personalidad puedan tener una incidencia interactiva de otro tipo (Garanto et al., 1985).

\section{SÍNTESIS DE RESULTADOS Y COMENTARIOS}

Los resultados recogidos en los apartados anteriores son coincidentes en determinados casos, con algunas de las tendencias más abiertamente resaltadas por la investigación en otros paises, pero no lo son en todos. Algunos de los hallazgos empíricos no refuerzan los más comunes en investigaciones especializadas en otros contextos.

La preparación académica previa de carácter común de los estudiantes de áreas científico-tecnológicas no parece ser muy diferente de la que ofrecen los estudiantes de otras áreas universitarias. En todo caso, se puede hablar de inexistencia de una tendencia sistemática de diferenciación, aunque, en conjunto, los alumnos de Ciencias e Ingeniería parecen ser algo seleccionados en cuanto a preparación previa común.

Se observan diferencias entre las muestras de varones y mujeres de forma que, éstas tienden a destacar en las pruebas de acceso, mientras que los valores lo hacen en la prueba de madurez. Las mujeres de ciencias son de muy alto rendimiento en cuanto a la parte común de la selectividad, mientras que los varones del área científico-tecnológica destacan especialmente en las medidas de madurez académica.

Se confirma el carácter predictivo de la preparación previa sobre el rendimiento académico universitario. Este hecho es general para todo tipo de estudios.

Las correlaciones parecen estar condicionadas por el sexo y probablemente lo están de manera algo diferente en las carreras de ciencias que en otras carreras. Sin embargo, resulta aventurado establecer generalizaciones al respecto.

Nuestros datos parecen confirmar el extendido resultado de que el ahumnado de carreras científicotecnológicas es bastante seleccionado desde el punto de vista de aptitud, sobre todo en el razonamiento numérico, el razonamiento abstracto y la inteligencia general.

En buena parte de las variables, esta selección de aptitud parece ser algo más rigurosa, en términos relativos, entre las mujeres que entre los varones.

Las correlaciones de las aptitudes con el rendimiento universitario son, en general, poco significativas en todos los tipos de estudio y en ambos sexos, aunque se atisba la posibiliđad de que la comprensión verbal sea un predictor significativo del rendimiento en los varones y el razonamiento numérico lo sea en las mujeres.

Efectivamente, nuestros estudiantes de ciencias tienen cierta tendencia a la introversión, pero no más que los estudiantes de otras carreras, como podría desprenderse de algunas sintesis de la investigación. Tampoco se observan diferencias claras entre los diferentes estudios en cuanto a otros rasgos de personalidad. Donde se encuentran algunas diferencias es entre varones y mujeres, en algunos rasgos, pero no son tendencias claras y sistemáticas.

El último hallazgo confirma la creencia de los investigadores de que entre el rendimiento y los rasgos de personalidad no existe una relación lineal y que, en todo caso, debe hablarse de otro tipo de interacción.

Nuestros resultados aportan nuevos datos para una linea de estudio e investigación establecida y reconocida entre los especialistas en enseñanza de las ciencias, pero también pueden ser un elemento referencial para la planificación y la evaluación didáctica en las clases universitarias de ciencias. Así, por ejemplo, una extrapolación de alguno de los anteriores resultados nos lievaria a pensar que el alto indice de fracaso universitario en los estudios cientifico-tecnológicos no parece ra- 
zonable que pueda estar causado por problemas de aptitud, ya que estos alumnos son, comparativamente hablando, selectos desde este punto de vista y potencialmente capaces. Por tanto, el profesorado de estas carreras debe reflexionar seriamente sobre este hecho.

Sin embargo, sin cuestionar la validez de nuestros resultados y de la anterior reflexión, hay que reconocer que existe otra línca vigente de trabajos de investigación que se articula alrededor de un hecho aparentemente contradictorio con nuestros hallazgos; los deficientes niveles de razonamiento y de desarrollo cognitivo son causa fundamental en el alto nivel de fracaso de los alumnos en los primeros cursos de áreas cientificas. En consecuencia, el desarrollo de niveles de razonamiento se convierte en un objetivo básico a conseguir en los primeros cursos de carreras científicas (Braga 1987).

\section{REFERENCIAS BIBLIOGRÁFICAS}

ABRAHAM, M.R., RENNER, I.W., GRANT, R.M. y WESTBROOK, S.L., 1982. Priorities for research in science education: A survey, Journal of Research in Science Teaching, Vol. 19 (8), pp. 697-704.

BAKER, D.R., 1985. Predictive value of attitude, cognitive ability, and personality to science achievement in the middle school, Journal of Research in Science Teaching, Vol. 22 (2), pp. 103-113.

BENBOW, C.P. y MINOR, L.L., 1986. Mathematically talented males and females and achievement in the high school sciences, American Educational Research Journal, Vol. 23, (3), pp. 425-436.

BRAGA, I.L., 1987. iIngresan los alumnos en la universidad con un adecuado desarrollo de los niveles de razonamiento? Enseñanza de las ciencias, Vol. S, (1), pp. 16-26.

BUTTS, D., CAPIE, W., FULIER, E., MAY, D., OKEY, J. y YEANY, R., 1978. Priorities for researh in science education: a delphi study, Journal of Research in Science Teaching, Vol. 15 (2), pp. 109-114.

COLLINGS, J. y SMITHERS, A., 1984, Person orientation and science choice, European Journal of Science Education, Vol. 6 (1), pp. 55-65.

DEBOER, G.E., 1986. Perceived science ability as a factor in the course selections of men and women in college, Journal or Research in Science Teaching, Vol. 23, (4), pp. 343-352.

ESCUDERO, T., 1985. Las actitudes en la enseñanza de las ciencias: un panorama complejo, Revista de Educación. Vol. 278, pp. 5-25.

ESCUDERO, T., 1986. Seguimiento a la selectividad universitaria. (Investigación 19, ICE-Universidad de Zaragoza).
Ante ambas perspectivas, no existe duda de que nos encontramos con un problema de graves implicaciones curriculares en la enseñanza de carreras científicotecnológicas, que recogen a buena parte de los alumnos más capaces, pero también son unos estudios con unos niveles de fracaso escolar comparativamente muy alto, en general en gran medida causado por el escaso nivel de razonamiento inicial de buena parte de los alumnos. El conflicto y sus consecuencias - abandono progresivo de los estudios científico-tecnológicos por parte de los alumnos - no tendrá solución si no se acomete con seriedad un análisis critico y una profunda revisión de los niveles iniciales de tales estudios universitarios y de su coordinación con los estudios previos, lo que no tiene por qué implicar un descenso en la calidad y en los niveles de preparación de los futuros graduados, sino más bien una mejora global en la formación del conjunto de nuestros titulados.

ETHINGTON, C.A. y WOLFLE, L.M., 1986. A structural model of mathematics achievement for men and women, American Educational Research Journal, Vol. 23, (1), pp. $65-75$.

GARANTO, J., MATEO, J. y RODRÍGUEZ, S., 1985. Modelos y técnicas de análisis del rendimiento académico, Revista de Educación, Vol. 277, pp. 127-169.

HACKER, R.G., 1986. Class gender and science teaching processes, European Joumal of Science Education, Vol. 8 (1), pp. $57-71$.

HOLLAND, J., 1973. Making vocational choices: $A$ theory of careers. (Prentice-Hall, Englewood Cliffs, N.J.).

LOWERY, L.F., 1980. Toward improving the science education researh cnterprise, Journal of Research in Science Teaching, Vol. 17, (4), pp. 275-281.

MARASCUI1.O, L.R., 1971. Statistical Methods for Behavioral Science Research. (McGraw Hill: New York).

SACHS, L., 1978. Estadística aplicada. (Labor S.A: Barcelona).

STEINKAMP, M.W. y MAEHR, M.L., 1983. Affect, ability, and science achievement: A quantitative synthesis of correlational research, Review of Educational Research, Vol. 53, pp. 369-396.

WELCH, W.W., 1985. Research in science education: Review and recomendations, Science Education, Vol. 69, (3), pp. $421-448$.

YAGER, R.E. y PENICK, I.E., 1987. Resolving the crisis in science education: understanding before resolution, Science Education, Vol. 71, (1), pp. 49-55. 